\title{
Image Compression and Plants Classification Using Machine Learning in Controlled-Environment Agriculture: Antarctic Station Use Case
}

\author{
Sergey Nesteruk, Dmitrii Shadrin, Mariia Pukalchik, Andrey Somov, Conrad Zeidler, \\ Paul Zabel, and Daniel Schubert
}

\begin{abstract}
In this article, we share our experience in the scope of controlled-environment agriculture automation in the Antarctic station greenhouse facility called EDEN ISS. For remote plant monitoring, control, and maintenance, we solve the problem of plant classification. Due to the inherent communication limitations between Antarctica and Europe, we first propose the image compression mechanism for the data collection. We show that we can compress the images, on average, 7.2 times for efficient transmission over the weak channel. Moreover, we prove that decompressed images can be further used for computer vision applications. Upon decompressing images, we apply machine learning for the classification task. We achieve $92.6 \%$ accuracy on an 18-classes unbalanced dataset. The proposed approach is promising for a number of agriculture related applications, including the plant classification, identification of plant diseases, and deviation of plant phenology.
\end{abstract}

Index Terms-Classification, computer vision, controlledenvironment agriculture, image compression, machine learning

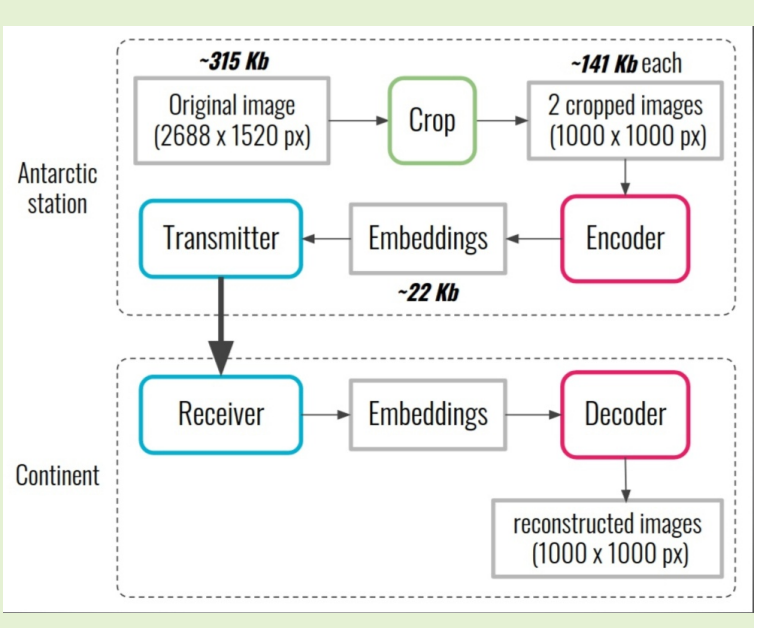

\section{INTRODUCTION}

$\mathbf{C}$ ONTROLLED-environment Agriculture (CEA) is an approach based on the state-of-the-art technologies involved in food production. Indeed, recent evolving of the Internet of Things (IoT), Artificial Intelligence (AI), and Robotics [1] [2] foster development of CEA and consequent appearance of vertical farms, greenhouses, and hoophouses [3]. IoT and Robotics serve to automate the data collection and actuation proceses [4] [5], while AI enriches them with intelligent capabilities [6]. It should be noted that CEA is applied for food production in space for providing the aeronauts with fresh food [7]. At the same time, CEA is vital for the same reason on Earth: food production in remote areas, e.g., Antarctic, could address the problem of regular food provisioning.

Although there are myriad research papers reporting on the progress of the technologies mentioned earlier, just a small portion is targeted for the Agriculture domain. Moreover,

S. Nesteruk, D. Shadrin, M. Pukalchik, A. Somov are with the Center for Computational and Data-Intensive Science and Engineering (CDISE), Skolkovo Institute of Science and Technology, 121205 Moscow, Russia.

C. Zeidler, P. Zabel, D. Schubert are with the Institute of Space Systems, German Aerospace Center (DLR), Bremen, Germany. there is a lack of research reporting on the pilot studies and deployments in real settings, which could shed the light on how to address a number of practical problems in CEA. To make the research practically feasible, some of the developed methods were tested in lab conditions [8].

In this article, we share our experience in CEA on the EDEN ISS experimental facility located at the Neumayer Station III site in the Antarctic. EDEN ISS is aimed at advanced life support research in the remote area. This facility includes the Mobile Test Greenhouse, which is devoted to autonomous cultivation for more than thirty higher plant species. The motivation for this Mobile Test Greenhouse has its roots in the plan for future human exploration missions. The driving objective for the EDEN ISS project is a successful autonomous operation where crops must be grown to maturity level. For effectuating the remote control of plant growth, we need to send the images from the South Pole to Europe continuously. This activity is constrained by the limitation of the communication system allowing for the transmission of just a few images per day. That is why, before beginning the data analysis, we have to address the problem of dataset collection. For this reason, we first propose an image compression method followed by solving the problem of plant classification using machine learning applied to the collected dataset. 
The novelty of this work is twofold: (i) we address the problem of image compression and further plant classification in a real agriculture scenario characterized by the system limitations, (ii) we performed the image compression using HiFiC model [9] with a 7.2 compression ratio.

This article is organized as follows: we introduce the reader to the state-of-the-art in image compression and image analysis in Section II. Next, we discuss the research scenario and challenges in Section III. Methods used in this work are described in Section IV. We then present the data analysis in Section V. We address the problem of plant classification using the images collected and decompressed and applying machine learning. Finally, we provide concluding remarks in Section VI.

\section{RELATED WORKS}

\section{A. Image Analysis for Plant Phenotyping}

Many methods of plant phenotype measuring require to uproot or cut a plant. These techniques either claim very specific cultivation conditions [10] or kill plants and make it impossible to track plants' phenotype change in time. Therefore it is vital to have non-destructive methods for automated plant phenotyping [11]. Popular non-invasive plant phenotyping methods are Magnetic Resonance Imaging (MRI) [12], Computer Tomography $(\mathrm{CT})$, Position Emission Tomography (PET) [13] and and multispectral imaging [14]. It is also difficult to widely implement the tools that imply costly equipment. Another promising approach is to use a $3 \mathrm{D}$ camera to make volumetric reconstruction on a plant. Such a technique is accurate and provides useful insights. However, they are difficult to use in practice. In the Antarctic station or further space missions, it becomes almost impossible to use such complex and huge machinery.

On the contrary, computer vision algorithms need relatively cheap 2D cameras to collect images of a plantation. They are easy to use and leave plants untouched [15]. Consequently, in our work, we focus on the algorithms that work with images. Images can be collected using regular cameras that are easy to install. It makes our solution easy to scale and very robust.

In recent years we see an increase of the research activity in the field of noninvasive PP [16] [17]. To conduct successful $\mathrm{PP}$ research, we usually have to collect much data [18]. To automate the data collection process, we usually use various Internet of Things (IoT) systems. They not only speed up the overall process but also allows us to collect data more often and in a more homogeneous way. Furthermore, it reduces the number of routine tasks that qualified agronomist or biologist has to perform, freeing up time for more advanced doings [19].

\section{B. Image Compression}

A considerable amount of images is produced every day for various applications, and for the plant phenomics in particular. Image data contains a large amount of useful information, spatial characteristics of the investigated object that cannot be described in any other way. However, this type of data requires much space [20]. To store and transmit them efficiently we need robust image compression techniques. That makes it a popular research area for decades.

All image compression algorithms can be split into two categories: lossless compression and lossy compression. Lossless compression algorithms do not lose any information and recover every bit of the original image [21]. Lossy compression algorithms eliminate redundant information that cannot be recovered back [22] [23] [24]. The goal of lossy compression is to reduce the size of the input image dealing with non-crucial perceptual changes to it. Usually, we can vary the level of acceptable changes that will also affect the size of the reduced image. Lossy compression decreases the image size better. Moreover, some techniques for lossless compression, such as entropy coding, can be used with lossy compression [25].

In this work, we apply the lossy compression since our data is not too sensitive for minor changes, and it is crucial to get higher compression level limitations in the transmission channel. The most widely used lossy compression codec today is JPEG [22]. Other algorithms that gain popularity are JPEG200 [23] and WebP [24]. Another fundamentally different type of compression algorithms is based on neural networks. Convolutional networks learn representations and patterns from data, which allows us to use learned information about real objects to achieve higher compression [20] [26] [27] [28]. The cost of such performance is long compression and decompression time. Ten seconds for single image compression [9] is not suitable for Web applications; however, it is acceptable for transmitting data from the remote systems.

Currently, the models that are good in compression high-resolution images are based on Variational Autoencoders (VAEs) [26] [29], Recurrent Neural Networks (RNNs) [30] and Generative Adversarial Networks (GANs) [31]. VAEs learn partly invertible mappings from image pixels to a latent representation [32]. RNNs are designed to iteratively reduce the residual between the original image and the model's result at the previous iteration [33]. GANs consist of two networks that compete against each other during training [34]. The first generates an image, and the second tries to distinguish whether it is real or fake. This type of model is particularly good with high-resolution images.

\section{Scenario AND Challenges}

In this work, we address the problem of image compression and further plant classification in a real agriculture scenario characterized by system limitations and perspective to future human expansion to Mars. For this reason, a number of various plants are grown in the EDEN ISS facility located in Antarctic. Since it is a mainly unmanned facility, our primary goal is to ensure automatic maintenance for the plants. At the same time, to fully control the growing process and solve the plant classification task for the research reasons, we need to receive the images from the remote area to Europe continuously. Given the EDEN ISS facility is constrained in terms of data transmission from Antarctic to Europe and is able to send over a wireless channel just one image per day, we have 
proposed an image compression approach. Upon receiving the images, we decompress them and run the ML-based image classification.

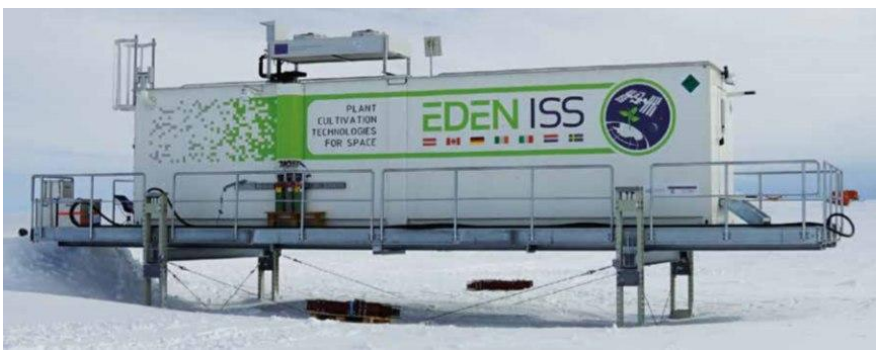

Fig. 1: EDEN ISS Mobile Test Facility.

The core of the EDEN ISS facility is Command, and Data Handling System (CDHS) performs several vital actions associated with data: in situ data acquisition, control, management, and storage within the Mobile Test Facility (MTF). Also, CDHS provides the remote access/control of the MTF from the Neumayer Station III (NM-III) and from User Home Bases (UHBs). CDHS is composed of two units:

- subsystem control and monitoring system,

- camera control system.

The EDEN ISS MTF is based on the commercial Argus platform. It guarantees the monitoring of the status of the MTF subsystems via telemetry as well as the interaction with them.

Configuration of video cameras is done using HIKVISION software. The cameras are controlled via the PC which is able to process the images from the cameras.

\section{METHODS}

\section{A. Hardware: Imaging System}

Image collection in the greenhouse is an essential task for guaranteeing the plants growth and health monitoring. The collected images provided the extensive datasets for further analysis and defining the following metrics: leaf size and color, morphology and development of plants, as well as leaf reflectivity values for improving the plant health assessments. This image collection system consists of two camera systems coordinated via a central network image processing server. The first camera system is the HD color image system, a set of remote-controlled, 4-megapixel, Red-Green-Blue (RGB) Webcams that monitor the entire greenhouse. The second camera system is two-wave spectral imagers, modified GoPro cameras taking images with the $12 \mathrm{MP}$ resolution. The objective of the second camera system is to study the applicability of difference-wavelength imaging for plant health monitoring.

The imaging system contains set of fixed high resolution cameras, organized in the following way (see Fig. 2 and Fig. 3). Seventeen digital cameras are located on the overlays and have top-down view and able to capture images of two trays. Seven digital cameras on the overlays of the L4 and have top-down view, each of these cameras is located above one tray, only the upper camera is aimed for taking images of all the level (see Fig. 2). Eight digital cameras are located in front of each rack on the opposite side and have the side view

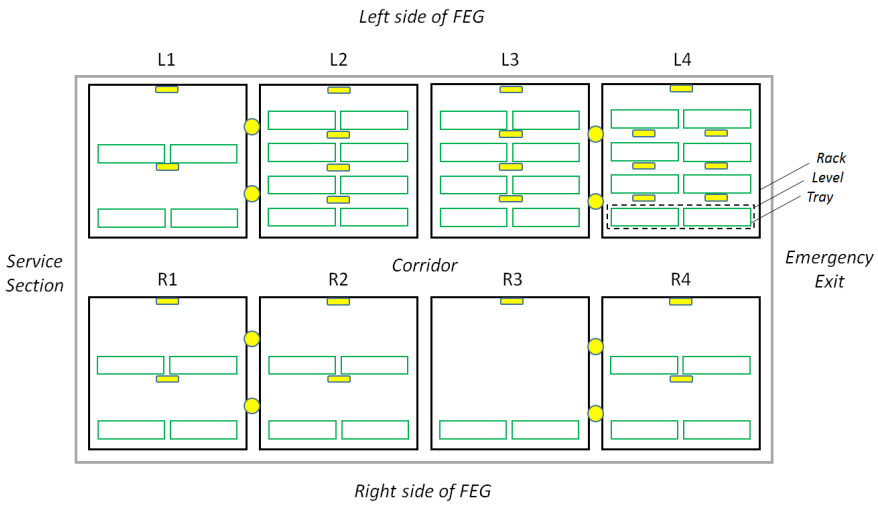

Fig. 2: Digital cameras location in the EDEN ISS. Black outline represents the plant growth racks (four) on each side of the growth module. R-right side, L-left side. Each rack contains four growth levels. Each growth level has two trays for plant cultivation. The yellow circles and squares represent the side and top view cameras, respectively.

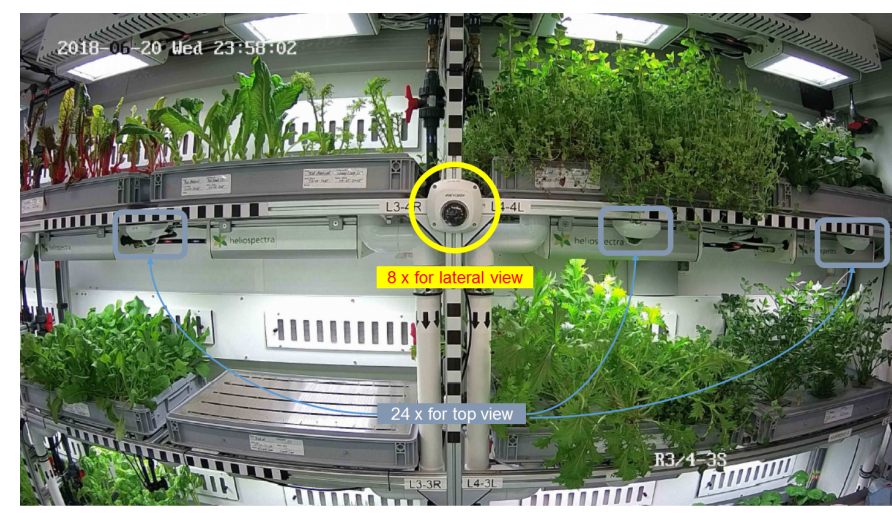

Fig. 3: Camera layout scheme example. Blue - top-view cameras; yellow - lateral cameras.

on the rack (see Fig. 3). All these cameras are connected to the local network run at the EDEN ISS. The entire hardware part is controlled by the software enabling the image collection, storage and transmission. The model of the high resolution cameras that was used is HIKVISION DS-2CD2542FWDI 4MP with the image resolution 2688 x 1520 pixels. The characteristics of cameras are the following: the focal length of the lens is $2.8 \mathrm{~mm}$, the view angle is $106^{\circ}$. Ethernet switches were used for connection of the MTF local network and connection was managed to the computer located in the module, where the special software HIKVISION for cameras management was installed. The camera system is organized in the way to have opportunity for remote control. The EDEN ISS operator has the options for making continuous streaming of images, video recording, and taking snapshots. The normal operation mode was defined as follows: taking one image from each camera per day. Images were saved in jpeg format and have maximum size $400 \mathrm{kB}$. This procedure was performed by custom software, developed in Python language. The reason for using the jpeg format for initial compression is the ability to send all the taken images to the mission control center 
located in Bremen. The connection is realized at low data rate $100 \mathrm{kbps}$. The developed software also allows for the convenient visualization of the obtained dataset, making it easy to monitor the current state of the plants in the EDEN ISS.

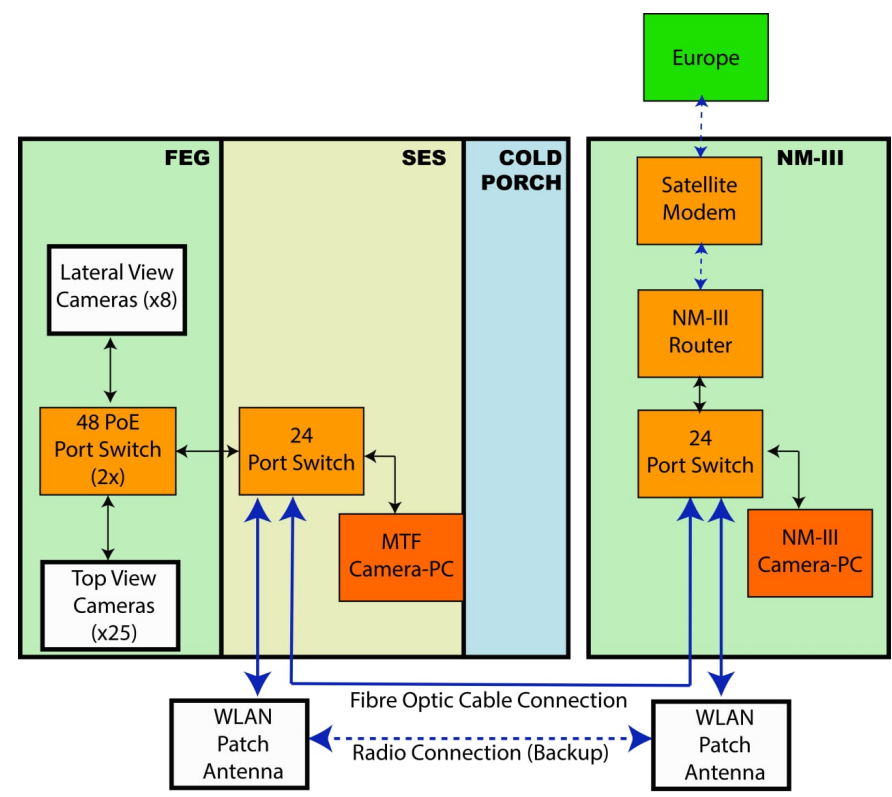

Fig. 4: Overall network layout between Antarctic station and continent. Black lines represent the local wired connections; solid blue lines are the fiber optic cable connection, dashed blue lines are radio connections. FEG is the greenhouse; SES is the service section; NM-III is Neumayer Station.

\section{B. Data Pre-processing}

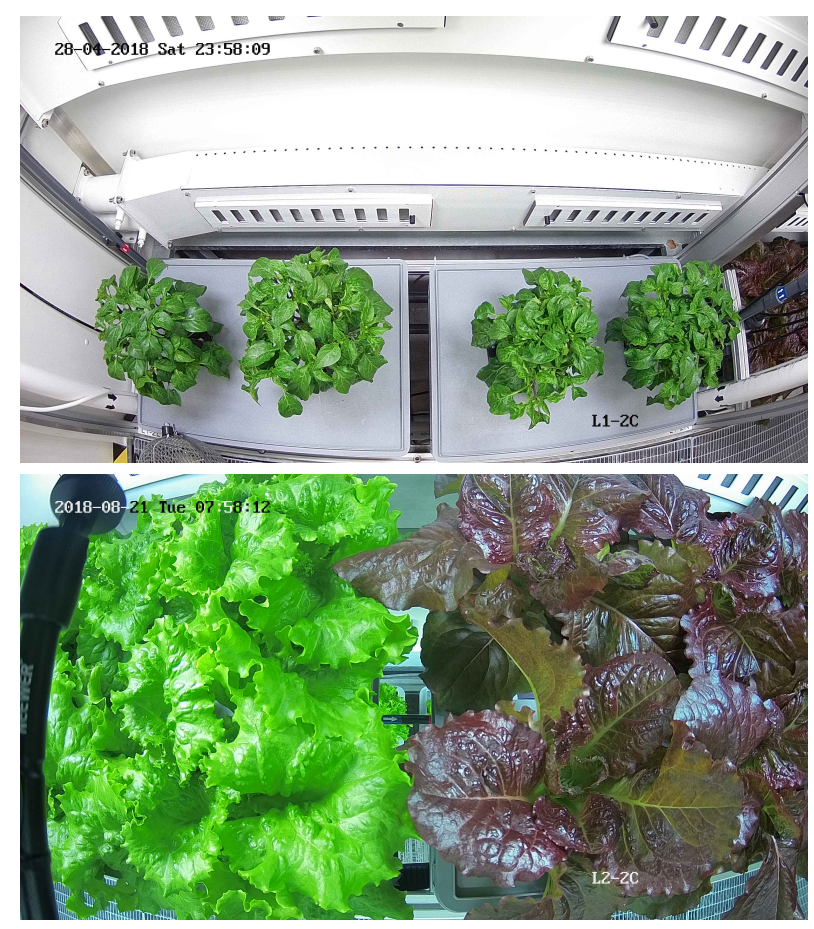

Fig. 5: Image samples from EDEN-ISS Antarctic dataset.
Our dataset consists of 6358 RGB top-view images of the plants in cultivation slots. The imaging rate is one frame per day with the imaging time at 12 p.m. The size of each image is $2688 \times 1520$ pixels. As we can see in Fig. 5, a single image can contain one or two different plant classes. In total, we have 17 plant classes and one special class for empty spots. The distribution of the data in classes is presented in Fig. 6. The classes are sorted in descending order for simpler visual comparison. It can be noticed that this is an imbalanced dataset, which in turn leads to the use of the most advanced ML and CV algorithms for processing such data to get an accurate result, for example, while solving a classification problem.

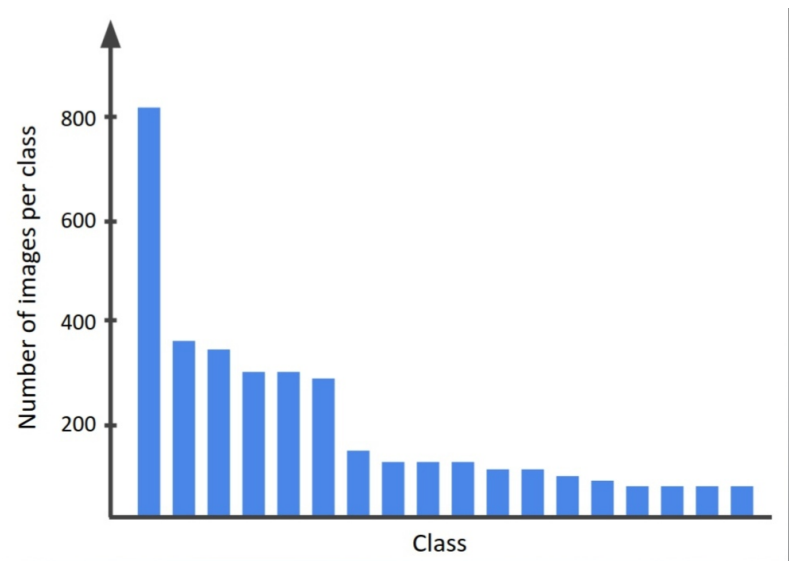

Fig. 6: Distribution of the classes in the dataset.

The first data pre-processing step is to filter out images that are not useful for neural networks. These are images with any obstacles that overlap plants, affecting the overall training process by adding false features to data. We also delete many images without plants at all because we do not need so many of them for training, thus making the dataset more balanced.

Non-empty images contain at least eight plants each. Usually, they have two plant varieties, on the left and right halves of the picture. To get unique plant classes on each image, we crop images on two halves. The resulting images are $1000 \times 1000$ pixels in size. Note that original images contain lines of text in the corners. It is essential to crop such auxiliary information because we do not want neural networks to "see" them. This ensures that models will not learn the features of the text instead of the features of plants.

After all the pre-processing steps, we have formed a dataset with 3184 images of 18 classes. The dataset is very unbalanced. To handle it, we use a balanced data loader. It operates by aligning the probability of getting a sample from each class. Our dataloader upsamples data to have an approximately equal number of samples of each class, which helps to avoid overfitting on the classes with the majority of images. Due to image augmentation, we also do not overfit on repeated images.

To make sure that the resulting model is not overfitted on the classes with the majority of samples, we add precision and recall metrics for the classification task.

After that, the dataset was divided into train, validation, and 
holdout subsets. The division is random and independent for each class. The proportion of the subsets is as follows: $60 \%$ for training, $20 \%$ for validating, and $20 \%$ for the holdout. The training subset is used to learn model parameters. The validation subset is used to check accuracy improvement after each learning iteration. The hold out subset is used after all the training to check final results. Such splitting reduces the number of samples available for training; however, it excludes overfitting on the training images and makes results much more reliable.

\section{Image Compression}

In this section, we show an approach for lossy image compression with neural networks. The general idea is to split a CNN into two independent parts. The first one (encoder) will perform calculations on the EDEN ISS station making tiny embeddings out of input images. The second part (decoder) will work on the continent and decompresses images back. The quality of an image will be slightly lower, but it will be enough for remote visual control of plant status and solving some plant phenotyping tasks in an automatic manner. The central trade-off in such a system is between the compression rate and the reconstructed image quality.

In our work, we apply the HiFiC [9] model for image compression. This model merges the ideas of Generative Adversarial Networks (GANs) and learned compression. The model consists of four networks: encoder $E$, hyper-prior [27] model $P$, generator $G$ and discriminator $D$. This model is trained on high-resolution images collected by its authors from the Internet and shows good results on CLIC2020 [35], Kodak [36], and DIV2K [37] datasets.

The authors of the original paper train several models of various target bpp rates. In our work, we choose the model with the highest compression rate. To compress an image, we pass it through an encoder, hyper-prior, and nonparametric entropy coder. Note that it enables embeddings to have different sizes. This feature makes it beneficial to use the described method compare to others. To reconstruct the image, we pass embeddings through the decoder of the entropy model and the generator network. The discriminator is used during the training to help the model obtain realistic outputs. The critical point is that the generator is trying to get realistic images close to the distribution of the original input, but it is not trained to get the exact reconstruction. but it's not trained to get the exact reconstruction.

Fig. 7 shows an example of image reconstruction. As we can see, the image loses quality near sharp edges. That makes it unsuitable for working with images of text, but good enough for the general purpose.

The average size of a cropped image in our dataset is $141 \mathrm{~Kb}$. After compression, the average size of embedding is $22 \mathrm{~Kb}$ with 0.24bpp (bits per pixel), which gives us 6.4 times benefit in file size. The bpp of every image varies depending on its complexity, but in the current model, it cannot be lower than $0.14 \mathrm{bpp}$.

We measure the quality of image reconstruction with PSNR and SSIM [38] metrics. The average PSNR on our data is 33.8. The average SSIM is 0.96 .
The approximate time for compression 1000x1000 pixels image is 3.5s on GPU. The decompression time is around 9s on GPU. As we can see, the model is asymmetric in terms of encoder-decoder computations. In the case of the Antarctic station, it is a huge benefit because the remote system has to spend fewer resources for compression.

\section{DATA ANALYSIS}

Upon collecting the images and preparing the dataset we perform the plant classification task. In this section, we show an approach that allows classifying the plant varieties. In further work, it can be extended for classifying the plant diseases, determination of the ripening stage, and finding anomalies.

Our solution is to apply the convolutional Variational AutoEncoders (VAE) [29]. The scheme is shown in Fig. 8. We encode initial data to get the latent representation and then feed it to the classifier. Note that in the VAE model, we can fix the length of an embedding, which makes it easier to use for auxiliary tasks.

We reflect the VAE classification results in Table I. All of the tested VAEs are based on ResNet34 [39] model. The choice is based on an intuition about required for this task model complexity. Embedding size is the size of image representation in latent space. It is the output of a VAE model and the input of a classifier. In other words, it is the size of the squeezed representation of the input image. A classifier is an algorithm that is used after the VAE. We use two types of classification algorithms for this task.

Support Vector Machine (SVM) is a linear model for the classification problem. It is known to work well for many practical problems. The idea of the SVM algorithm is to create a line or a hyperplane which separates the data into classes. To do so, SVM finds the points that are the closest to the border of a class (we call these points as support vectors) and searches the line that is as far as possible from the points.

XGBoost is a popular and effective implementation of a boosting algorithm. It works by aggregating the outputs of multiple weak tree-based models.

While model training, we augment images to increase the number of samples artificially. It allows feeding a model with various examples each epoch, thus avoiding overfitting. More precisely, we:

1) Normalize values of pixels separately for each channel.

2) Apply the colour jitter.

3) Apply random rotation for the angle from -30 to 30 degrees.

4) Apply the image horizontal flip in $50 \%$ on cases.

5) Apply 25\% dropout.

Normalization is performed by applying equation (1) for each image channel.

$$
C=\left(C-\text { mean }_{C}\right) / \operatorname{std}_{C}
$$

where $C$ is one of the RGB channels. The mean values are $(0.485,0.456,0.406)$ for red, green, and blue channels accordingly. The standard deviation values are $(0.229,0.224$, 0.225 ) for red, green, and blue channels accordingly. The 

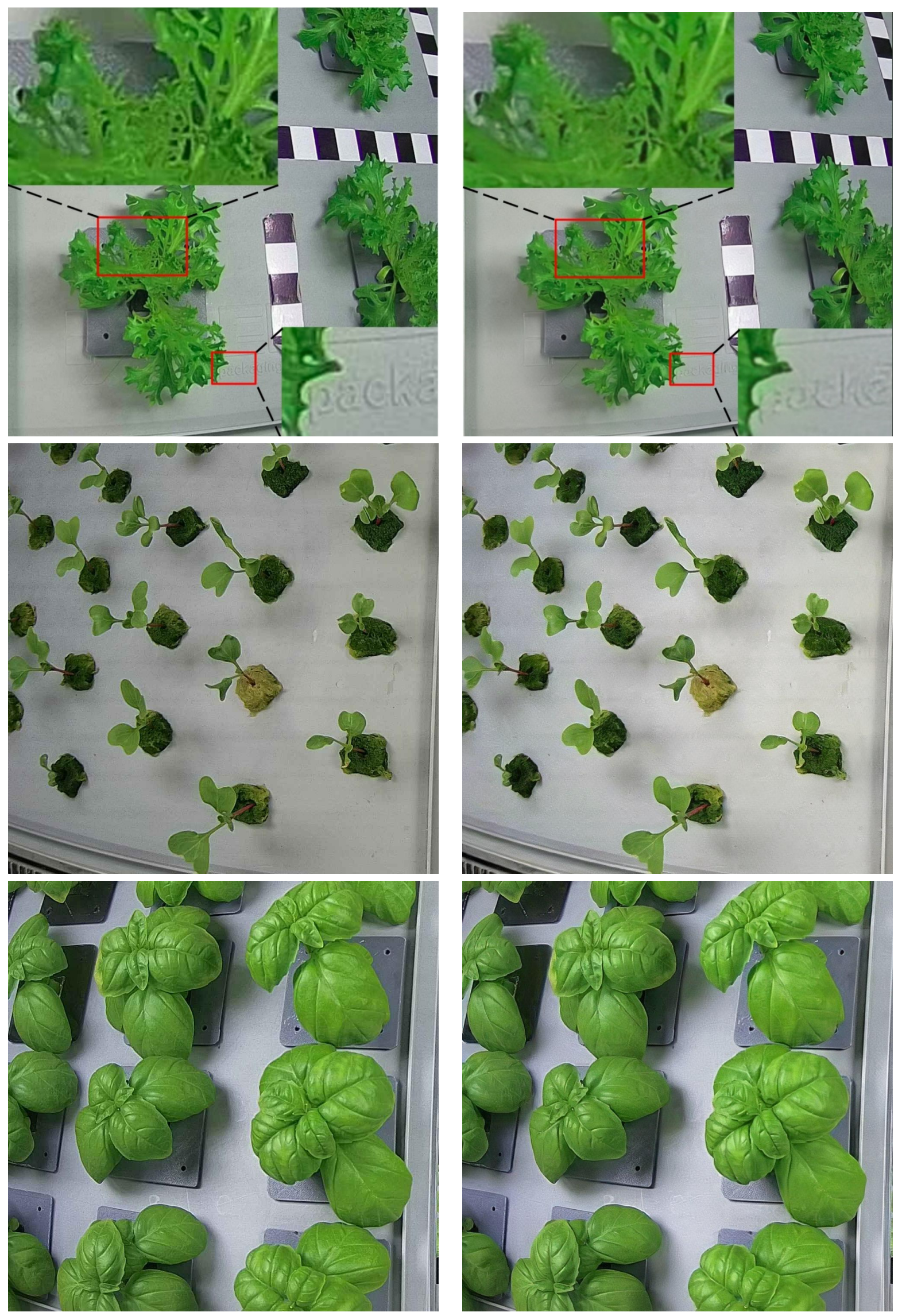

Fig. 7: Image compresion examples. Original images (on the left) and reconstructed images (on the right). 


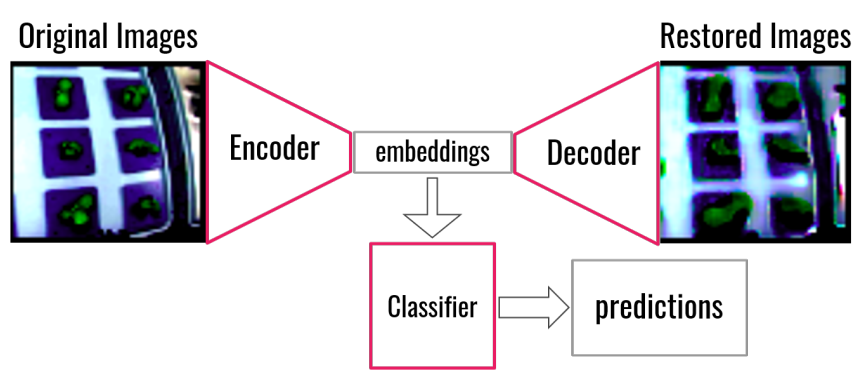

Fig. 8: The pipeline for classification with an autoencoder.

mean and standard deviation values for each channel are conventional for the general computer vision field and are obtained on large datasets.

Color jitter randomly changes the contrast and saturation of an image.

Random rotations increase the dataset semantic coverage. It works well because with convolutional networks an object and its rotated copy will result in slightly different feature maps.

Dropout sets some random values to zero. It adds noise and makes the model more robust.

All these steps allow for having more various samples for the training with the same amount of initial data.

The training setting for VAEs are:

- Total training epochs: 60 .

- L2 regularization factor: 0.05 .

- Initial learning rate: 0.001 .

- Exponential learning rate decay factor: 0.9 .

- Optimizer: Adam.

L2 is the regularization which adds an additional penalty (see equation (2)) to the initial loss function to avoid the overfitting.

$$
L_{\text {final }}=L+\lambda \sum_{i} \omega_{i}^{2}
$$

where $L_{\text {final }}$ is the final loss function, $L$ is the initial loss function, $\lambda$ is the weight decay factor, $\omega_{i}$ is the single weight value.

$$
l r_{e}=l r_{e-1} * \beta,
$$

where $l r_{e}$ is the learning rate at the $e^{t h}$ epoch, $\beta$ is the exponential learning rate decay factor.

Adam is a popular adaptive optimization algorithm in deep learning [40]. It fuses the ideas of RMSprop and SGD with momentum algorithms. More precisely, it uses the squared gradients to scale the learning rate, and it uses the moving average of the gradient. We use this optimizer as it usually converges fast on many computer vision tasks.

As we can see, the best performance shows a pipeline with 64 values sized embedding vector and SVM as a classifier. Fig. 9 demonstrates that the 2-dimensional representation of images is encoded to the latent space with 64 values embedding size. It shows how separable are our images before applying the classifier. We use the t-SNE algorithm to reduce a 64-dimensional vector to two dimensions. This transformation loses much information, that is why we use it only to visualize
TABLE I: Results of plants classification with VAE.

\begin{tabular}{|c|c|c|c|c|c|}
\hline Model & Accuracy & Precision & Recall & $\begin{array}{c}\text { Embedding } \\
\text { size }\end{array}$ & Classifier \\
\hline \multirow{5}{*}{ ResNet34 } & 0.8 & 0.81 & 0.8 & 20 & XGBoost \\
& 0.84 & 0.83 & 0.83 & 64 & XGBoost \\
& 0.82 & 0.82 & 0.83 & 128 & XGBoost \\
& 0.9 & 0.91 & 0.91 & 20 & SVM \\
& $\mathbf{0 . 9 2 6}$ & $\mathbf{0 . 9 3}$ & $\mathbf{0 . 9 2 7}$ & $\mathbf{6 4}$ & SVM \\
& 0.918 & 0.92 & 0.919 & 128 & SVM \\
\hline
\end{tabular}

the latent space. From this plot, we can learn which classes are harder to distinguish and estimate the overall performance of the encoder.

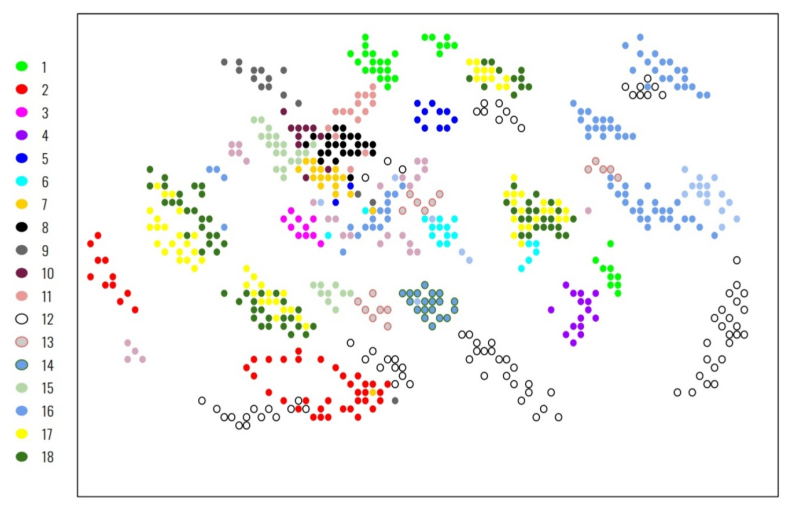

Fig. 9: 2D scatter plot of the embedding vector points using t-SNE. Dots with the same color contribute to the same class species.

\section{CONCLUSIONS}

In this article, we have demonstrated how machine learning methods are applied for solving real-world problems in Controlled-Environment Agriculture (CEA) domain. Our ultimate goal was to ensure the automation of a greenhouse and, in particular, to solve the plant's classification problem. We used images collected from the EDEN ISS facility located in Antarctic. Due to the communication limitations, we proposed an image compression method that helps compress the images with the ratio 7.2 and, therefore, receive more images per day. We achieved $92.6 \%$ accuracy on the 18-classes unbalanced dataset on the decompressed images.

The proposed approach based on the image compression and their transmission from Antarctic to Europe helps to solve a number of critical problems agriculture-related problems, including the CEA, e.g., classification, identification of plants diseases, deviation of plants phenology.

\section{ACKNOWLEDGMENT}

This work was supported by the Ministry of Science and Higher Education of the Russian Federation Agreement 07510-2020-091 (grant No 14.756.31.0001) and the European Union's Horizon 2020 research and innovation program (grant agreement No 636501 via the COMPET-07-2014 - Space exploration - Life support subprogramme). 


\section{REFERENCES}

[1] O. Elijah, T. A. Rahman, I. Orikumhi, C. Y. Leow, and M. N. Hindia, "An overview of internet of things (iot) and data analytics in agriculture: Benefits and challenges," IEEE Internet of Things Journal, vol. 5, no. 5, pp. 3758-3773, 2018.

[2] D. Shadrin, A. Menshchikov, D. Ermilov, and A. Somov, "Designing future precision agriculture: Detection of seeds germination using artificial intelligence on a low-power embedded system," IEEE Sensors Journal, vol. 19, no. 23, pp. 11573-11 582, 2019.

[3] A. Somov, D. Shadrin, I. Fastovets, A. Nikitin, S. Matveev, I. Oseledets, and O. Hrinchuk, "Pervasive agriculture: Iot-enabled greenhouse for plant growth control," IEEE Pervasive Computing, vol. 17, no. 4, pp. 65-75, 2018.

[4] R. Pahuja, H. K. Verma, and M. Uddin, "A wireless sensor network for greenhouse climate control," IEEE Pervasive Computing, vol. 12, no. 2, pp. 49-58, 2013.

[5] D. Shadrin, A. Somov, T. Podladchikova, and R. Gerzer, "Pervasive agriculture: Measuring and predicting plant growth using statistics and 2D/3D imaging," in Proceedings of the IEEE International Instrumentation and Measurement Technology Conference, 2018, pp. 1-6.

[6] H. Scharr, M. Minervini, A. French, C. Klukas, D. Kramer, X. Liu, I. Luengo, J. Pape, G. Polder, D. Vukadinovic, X. Yin, and S. Tsaftaris, "Leaf segmentation in plant phenotyping: a collation study," Machine Vision Applications, vol. 27, no. 4, pp. 585-606, 2016.

[7] K. Tomita-Yokotani, S. Anilir, N. Katayama, H. Hashimoto, and M. Yamashita, "Space agriculture for habitation on mars and sustainable civilization on earth," in 4th International Conference on Recent Advances in Space Technologies, 2009, pp. 68-69.

[8] D. Shadrin, A. Menshchikov, A. Somov, G. Bornemann, J. Hauslage, and M. Fedorov, "Enabling precision agriculture through embedded sensing with artificial intelligence," IEEE Transactions on Instrumentation and Measurement, vol. 69, no. 7, pp. 4103-4113, 2020.

[9] F. Mentzer, G. Toderici, M. Tschannen, and E. Agustsson, "High-fidelity generative image compression," in Advances in Neural Information Processing Systems, 2020, pp. 1-12.

[10] E. Adeleke, R. Millas, W. McNeal, J. Faris, and A. Taheri, "Variation analysis of root system development in wheat seedlings using root phenotyping system," Agronomy 2020, vol. 10, no. 2, p. 206, February 2020.

[11] D. Afonnikov, M. Genaev, A. Doroshkov, E. Komyshev, and T. Pshenichnikova, "Methods of high-throughput plant phenotyping for large-scale breeding and genetic experiments:," Russian Journal of Genetics, vol. 52, pp. 688-701, 072016.

[12] D. Pflugfelder, R. Metzner, D. Dusschoten, R. Reichel, S. Jahnke, and R. Koller, "Non-invasive imaging of plant roots in different soils using magnetic resonance imaging (MRI)," Plant Methods, vol. 13, no. 1, p. 102, November 2017.

[13] A. Wasson, K. Nagel, S.Tracy, and M. Watt, "Beyond digging: Noninvasive root and rhizosphere phenotyping," Trends in Plant Science, vol. 25, no. 1, pp. 119-120, January 2020.

[14] M. Minervini, H. Scharr, and S. A. Tsaftaris, "Image analysis: The new bottleneck in plant phenotyping [applications corner]," IEEE Signal Processing Magazine, vol. 32, no. 4, pp. 126-131, July 2015.

[15] W. Achim, F. Liebisch, and A. Hund, "Plant phenotyping: from bean weighing to image analysis," BioMed Central., vol. 1, pp. 1-11, August 2015.

[16] F. Fiorani, U. Rascher, K. Nagel, R. Pieruschka, S. Jahnke, M. Watt, and U. Schurr, "Application of plant phenotyping technologies for seed, leaf and root productivity traits." International Conference on Sustainable Agriculture and Bioeconomy, Feb 2017.

[17] C. Costa, U. Schurr, F. Loreto, P. Menesatti, and S. Carpentier, "Plant phenotyping research trends, a science mapping approach," Frontiers in Plant Science, vol. 9, p. 1933, 2019.

[18] S. M. S. da Cruz and J. A. P. do Nascimento, "Towards integration of data-driven agronomic experiments with data provenance," Computers and Electronics in Agriculture, vol. 161, pp. 14 - 28, 2019.

[19] F. Al-Turjman, "The road towards plant phenotyping via WSNs: An overview," Computers and Electronics in Agriculture, vol. 161, pp. 4 13, 2019.

[20] Z. Cheng, H. Sun, M. Takeuchi, and J. Katto, "Learning image and video compression through spatial-temporal energy compaction," in Proceedings of the IEEE/CVF Conference on Computer Vision and Pattern Recognition (CVPR), June 2019, pp. 10063-10072.

[21] B. Rusyn, O. Lutsyk, Y. Lysak, A. Lukenyuk, and L. Pohreliuk, "Lossless image compression in the remote sensing applications," in 2016
IEEE First International Conference on Data Stream Mining Processing (DSMP), 2016, pp. 195-198.

[22] G. K. Wallace, "The jpeg still picture compression standard," IEEE Transactions on Consumer Electronics, vol. 38, no. 1, pp. xviii-xxxiv, 1992.

[23] D. Taubman and M. Marcellin, JPEG2000 Image Compression Fundamentals, Standards and Practice. Springer US, 2013.

[24] G. Ginesu, M. Pintus, and D. D. Giusto, "Objective assessment of the webp image coding algorithm," Signal Processing: Image Communication, vol. 27 , no. 8 , pp. $867-874,2012$, special issue on: pervasive mobilemultimedia.

[25] W. Z. Wahba and A. Y. Maghari, "Lossless image compression techniques comparative study," Lossless Image Compression Techniques Comparative Study, vol. 3, no. 2, 2016.

[26] L. Theis, W. Shi, A. Cunningham, and F. Huszár, "Lossy image compression with compressive autoencoders," 2017.

[27] J. Ballé, D. Minnen, S. Singh, S. J. Hwang, and N. Johnston, "Variational image compression with a scale hyperprior," 2018.

[28] E. Agustsson, M. Tschannen, F. Mentzer, R. Timofte, and L. V. Gool, "Generative adversarial networks for extreme learned image compression," in Proceedings of the IEEE International Conference on Computer Vision, 2019, pp. 221-231.

[29] D. P. Kingma and M. Welling, "Auto-encoding variational bayes," 2013.

[30] G. Toderici, D. Vincent, N. Johnston, S. J. Hwang, D. Minnen, J. Shor, and M. Covell, "Full resolution image compression with recurrent neural networks," 2016.

[31] I. J. Goodfellow, J. Pouget-Abadie, M. Mirza, B. Xu, D. Warde-Farley, S. Ozair, A. Courville, and Y. Bengio, "Generative adversarial networks," 2014.

[32] D. Minnen, J. Ballé, and G. D. Toderici, "Joint autoregressive and hierarchical priors for learned image compression," in Advances in Neural Information Processing Systems 31, S. Bengio, H. Wallach, H. Larochelle, K. Grauman, N. Cesa-Bianchi, and R. Garnett, Eds. Curran Associates, Inc., 2018, pp. 10771-10 780.

[33] G. Toderici, S. M. O’Malley, S. J. Hwang, D. Vincent, D. Minnen, S. Baluja, M. Covell, and R. Sukthankar, "Variable rate image compression with recurrent neural networks," arXiv preprint arXiv:1511.06085, 2015.

[34] I. Goodfellow, J. Pouget-Abadie, M. Mirza, B. Xu, D. Warde-Farley, S. Ozair, A. Courville, and Y. Bengio, "Generative adversarial nets," in Advances in neural information processing systems, 2014, pp. 26722680.

[35] [Online]. Available: http://www.compression.cc/challenge

[36] R. W. Franzen. [Online]. Available: http://r0k.us/graphics/kodak

[37] E. Agustsson and R. Timofte, "Ntire 2017 challenge on single image super-resolution: Dataset and study," in Proceedings of the IEEE Conference on Computer Vision and Pattern Recognition (CVPR) Workshops, July 2017.

[38] Zhou Wang, A. C. Bovik, H. R. Sheikh, and E. P. Simoncelli, "Image quality assessment: from error visibility to structural similarity," IEEE Transactions on Image Processing, vol. 13, no. 4, pp. 600-612, 2004.

[39] K. He, X. Zhang, S. Ren, and J. Sun, "Deep residual learning for image recognition," 2015.

[40] D. P. Kingma and J. Ba, "Adam: A method for stochastic optimization," 2014.

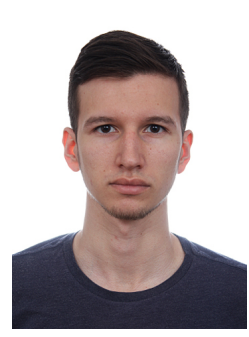

Sergey Nesteruk is a PhD student at the Skolkovo Institute of Science and Technology (Skoltech), Russia. Sergey received his BS and MS in Information Security at Saint Petersburg University of Aerospace Instrumentation in 2018 and 2020, respectively. In 2020 he also received his MS degree in Information Science and Technology at Skoltech. Sergey's research is related to monitoring systems and applying Machine Learning methods to the collected data. Sergey is involved in the development of Precision Agriculture Lab at Skoltech and is responsible for the development of greenhouse image collecting systems and Computer Vision research. 


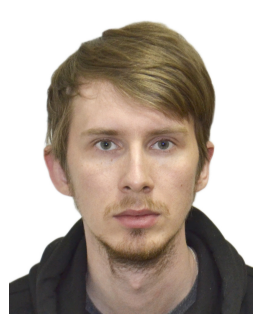

Dmitrii Shadrin is a Researcher at the Skolkovo Institute of Science and Technology (Skoltech), Russia. Dmitry received his PhD in Data Science from Skoltech in 2020 and MS in Applied Physics and Mathematics at the Moscow Institute of Physics and Technology (MIPT) in 2016. His research interests include data processing, modelling of physical and bio processes in closed artificial growing systems, machine learning, and computer vision. Dmitry is involved in the development of Digital Agriculture Lab at Skoltech and is responsible for the experimental research and a number of projects in the lab.

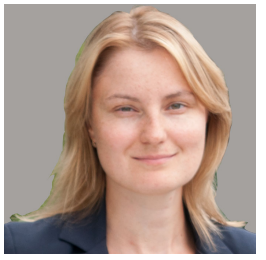

Mariia Pukalchik received the M.Sc. degree in ananlytical chemistry from the Vyatka State University, Russia, in 2009, and the Ph.D. degree in Ecology from the Lomonosov Moscow State University (MSU), Russia, in 2013. In 2017, she joined the Skoltech, where she is currently an Assistant Professor. She has authored 20 refereed articles and two books. Maria has experience in the field of of Machine Learning and Artificial intelligence application in biomedical, environmental, and agriculture fields. Mariia is involved in the development of Digital Agriculture Lab at Skoltech.

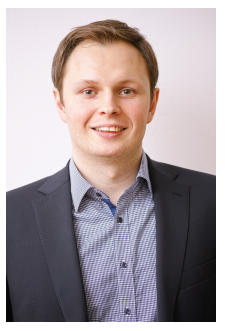

Andrey Somov is an Assistant Professor at Skolkovo Institute of Science and Technology (Skoltech), Russia. He graduated at 'MATI'Russian State Technological University, Russia (2004) and holds the diploma of Electronics Engineer from the same institution (2006). Andrey received his $\mathrm{PhD}$ (2009) from the University of Trento, Italy, for work in the field of power management in wireless sensor networks (WSN). Before joining Skoltech (2017), he had worked as a Senior Researcher for FBK CREATE-NET Research Center, Italy (2010-2015) and as a Research Fellow for the University of Exeter, UK (2016-2017). Andrey has published more than 80 papers in peer-reviewed international journals and conference proceedings. His current research interests include intelligent sensing, machine learning, cognitive loT and associated proof-of-concept implementation. Dr. Somov holds some awards in the fields of WSN and IoT including the Google IoT Technology Research Award (2016) and the Best Paper Award at IEEE IoP conference (2019).

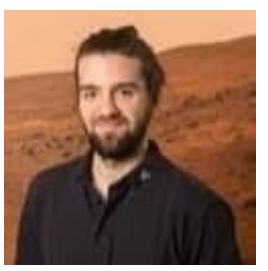

Conrad Zeidler graduated in 2011 at the Tech nical University of Brunswick, Germany, in Industrial Engineering majoring in Aerospace Engineering. Since 2012 he is a scientific employee at the Institute of Space Systems of the German Aerospace Center (DLR) in Bremen, Germany, in the Department System Analysis Space Segment. As a member of the EDEN research group he is responsible for monitoring and controlling plant and environmental parameters in the space habitation plant laboratory of the DLR in Bremen, Germany. In addition he is the manager of the mission control center for the EDEN ISS project including interaction with the operators of the MTF in Antarctica as well as the worldwide horticultural and engineering backrooms. In the frame of the EDEN ISS project he went several times to Antarctica to work on the EDEN ISS greenhouse during the maintenance deployment mission during summer season.

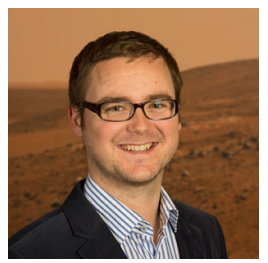

Paul Zabel has been working at the DLR Institute of Space Systems in the Space Segment Systems Analysis department since 2001. He has a degree in Aerospace Engineering from the Technical University of Dresden, Germany. $\mathrm{He}$ works in the EDEN research group, founded in 2011. His research area is life support systems for future crewed space missions. He has specialised in the development of plant cultivation systems for food production during such missions. Since 2015, Zabel has been working on the international EDEN ISS project aiming at the testing of technologies for plant cultivation in space.

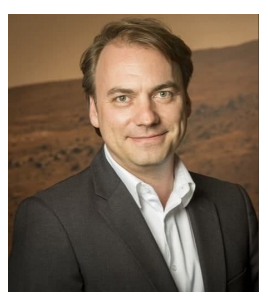

Dr.-Ing. Daniel Schubert studied at the Technical University of Berlin and has an engineering diploma in industrial engineering with an emphasis on aerospace and production techniques. In 2011, he initiated the EDEN group at the DLR Institute of Space Systems for technology investigations on Bio-regenerative Life Support Systems and since served as the team leader of this group. His research expertise is set on habitat interface analysis and plant accommodation and dynamic plant production planning. Throughout many projects for ESA, EU, Bundesministerium für Bildung und Forschung (BMBF), Wirtschaftsförderung Bremen (WfB), Dr. Schubert proved his management- and team leading skills. Outstanding is the EDEN ISS project. He led this project with 15 international partners, including the organization of the deployment mission of the greenhouse system at the Antarctic research station Neumayer III in 2017/18. 